\title{
Genetische Analyse zweier Mutanten von Dinophilus gyrociliatus (Archiannelida) mit veränderter Eigröße
}

\author{
W. Traut \\ Zoologisches Institut der Universität des Saarlandes, Saarbrücken
}

\begin{abstract}
Genetic analysis of two mutants of Dinophilus gyrociliatus (Archiannelida) with changed egg-size. D. gyrociliatus has two types of eggs: a smaller type giving rise to $\hat{\delta} \hat{b}$, and a larger type developing into $q$ ㅇ. Two mutants, which originated in laboratory cultures derived from animals collected at Helgoland, produced eggs of an abnormal size; the first, " $G r$ ", yielding larger-than-normal male-type eggs, the second, " $K l$ ", smaller male-type eggs. Size of eggs is determined by the genotype of the mother, the genotype of the eggs being without influence. Both mutants show a dominant monofactorial inheritance. In crosses with $G r / G r$-individuals an exceptionally high rate of reverse mutations to wildtype (1,4 to $5,0 \%$ ) has been observed. Results indicate that $G r$ is either an unstable gene or a chromosome mutation in the form of a duplication or trisomy of one chromosome.
\end{abstract}

\section{EINLEITUNG}

Dinophilus gyrociliatus $(=D$. apatris) erzeugt zwei verschieden große Sorten von Eiern (KoRschelt 1882). Aus der großen Eisorte entstehen im Normalfall $q$, aus der kleinen Eisorte $\hat{\delta} \hat{o}$. Die beiden Eisorten, die man demgemäß auch als $\hat{\delta}-\mathrm{bzw}$. O-Eier bezeichnet, werden in einem durch Umwelt und Genotyp der Mutter bestimmten Verhältnis abgelegt (Traut 1967).

In Zuchten, die zur Klärung des Geschlechtsbestimmungsmechanismus angesetzt worden waren, traten zwei Mutanten von D. gyrociliatus auf, die in Kultur gehalten werden konnten. Ihr hervorstechendes Kennzeichen ist eine gegenüber dem Normalzustand veränderte Größe der $\hat{\delta}$-Eier. Bei der zuerst aufgetretenen Mutante, die als "Größere §-Eier" bezeichnet werden soll (Symbol „Gr"), sind die $\hat{\delta}$-Eier vergrößert und liegen in der Größe etwa intermediär zwischen den Q - und $\partial$-Eiern des WildtypStammes (vgl. Abb. 3). Bei der zweiten Mutante ist das auffälligste Merkmal eine Verkleinerung der $\hat{\delta}$-Eier gegenüber denen des Wildtyp-Stammes (vgl. Abb. 3). Die Bezeichnung dieser Mutante ist „Kleinere ô-Eier“ (Symbol „Kl“).

Über die Auswirkungen der veränderten Eigröße auf die Geschlechtsentwicklung in Stamm Gr wurde bereits kurz berichtet (Traur 1966a). In der vorliegenden Untersuchung soll der Erbgang dieser beiden ersten bei Dinophilus gefundenen Mutanten analysiert werden. 


\section{MATERIAL UND METHODEN}

Für die Kreuzungsexperimente wurde neben den Mutanten-Stämmen, deren Ableitung aus einem Helgoländer Stamm bei den Ergebnissen beschrieben wird, ein Wildtyp-Stamm aus Helgoland [Stamm: A III (normal)] benutzt.

Die $q 9$ wurden einzeln in Schälchen von 1 bis $1,5 \mathrm{ml}$ Fassungsvermögen in pasteurisiertem Seewasser gehalten. Alle 5 Tage wurden sie in frische Schälchen umgesetzt und mit einer einzelligen Alge (Pseudostichococcus (?) monallantoides L. MoEwus) gefüttert. Die Zuchten standen in einem klimatisierten Raum bei $20^{0} \pm 1 / 2^{0} \mathrm{C}$ und 12-Stunden-Tag. Um jegliche Verschleppung zu vermeiden, wurden für jedes Tier und jeden Arbeitsgang frische sterile Pipetten benutzt.

Die Jungtiere schlüpfen unter den gegebenen Bedingungen am 6. Tag aus den

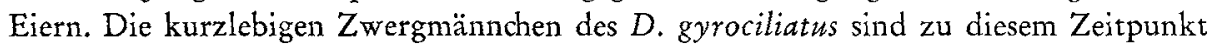
schon geschlechtsreif und kopulieren sofort mit den noch juvenilen, frisch geschlüpten 우우 oder mit älteren 우우. Die 우우 bewahren ihren Spermienvorrat bis zur Eiablage auf, die etwa am 6. Tage nach dem Schlüpfen beginnt. Die Generationsdauer beträgt also etwa 12 Tage. Zur Kreuzung isoliert man die $q q$ vor dem Schlüpfen noch in der Eihülle aus dem Eikokon und führt sie nach dem Schlüpfen mit dem gewünschten, möglichst frisch geschlüptten $\hat{\delta}$ bis zur erfolgreichen Kopulation zusammen (TRAU'T 1966b).

$\mathrm{Da}$ die durchschnittliche Größe der abgelegten $\hat{\delta}$-Eier als entscheidendes Merkmal den Phaenotyp eines $O$ charakterisiert, müssen zu seiner Feststellung alle $ㅇ ㅜ$ bis zur Ablage einer genügenden Zahl von Eiern isoliert gehalten und deren abgelegte Eier ausgemessen werden. Zunächst wurden bei allen $\$ \circ$ die Eier über einen Zeitraum von 20 Tagen ausgezählt und ausgemessen, später erwiesen sich 5 bis 10 Tage als ausreichend. Gemessen wurde der jeweils kleinste Eidurchmesser (die „Breite") mit Hilfe eines Okularmikrometers. Entscheidend für die Zuordnung zu den Phaenotypen "Gr",

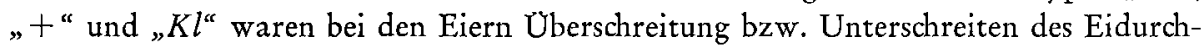
messers von $34 \mu \mathrm{m}$ und $42 \mu \mathrm{m}$. Lagen die Werte in der Nähe des Grenzbereiches, wurden die zugeordneten Individuen als „Gr?" bzw. „+?“ oder „Kl? " gezählt. Zu berücksichtigen ist noch, daß die Eier im ersten abgelegten Kokon immer kleiner als in den folgenden sind und daher nicht mitgezählt werden düfen. Naturgemäß lassen sich nach diesem Merkmal nur die 우 unterscheiden. Die Aufspaltungsergebnisse in den Tabellen beziehen sich daher nur auf die Töchter. Die Söhne müssen unberücksichtigt bleiben.

\section{ERGEBNISSE}

$$
\text { Gr-Mutante ( "Größere } \sigma^{\nearrow} \text {-Eier“) }
$$

Vergrößerte $\hat{\delta}$-Eier waren in der 15. Inzuchtgeneration in dem Helgoländer Stamm A III bei einem einzigen $q$ aufgetreten. Von diesem $q$ leiten sich alle später verwendeten $G r$-Tiere ab. Die Mutation wurde in den Helgoländer Stämmen A I und A III ein einziges Mal unter rund 2300 kontrollierten Einzelzuchten des NormalStammes beobachtet. In dem Pazifik-Stamm D wurde eine Mutante mit demselben Phaenotyp unter 850 Einzelzuchten gefunden. Dieser Fall konnte nicht näher untersucht werden, da die Linie nach einigen Generationen ausstarb. Es ließ sich aber wenig- 
stens feststellen, daß der Erbgang dominant war. In den 350 kontrollierten Zuchten der ïbrigen Wildtyp-Stämme wurde keine weitere $G r$-Mutante gefunden.

$U \mathrm{~m}$ einen reinerbigen $G r$-Stamm zu erhalten, wurden für die Weiterzucht über 43 Generationen hinweg nach Möglichkeit nur solche Tiere ausgewählt, die aus einer nichtspaltenden reinen $G r$-Nachkommenschaft stammten. Reinerbigkeit des Stammes wurde dabei jedoch nicht erreicht. Unter 781 kontrollierten $+9+$ mit ausreichenden Eiablagen waren $11,9 \%+$ ? - oder +-Tiere. Bei strenger Selektion von der 15. bis zur 34. Generation verringerte sich zwar der Anteil der +?- und +-Tiere, sie traten jedoch immer wieder vereinzelt auf $(3,7 \%)$. Bei Nachlassen der scharfen Selektion in der 35. Generation erhöhte sich der Anteil der + -Tiere sehr schnell wieder.

\section{Kreuzungen zwischen Gr-und Normal-Stamm}

In ersten Kreuzungen zeigte sich, daß man mit einem monohybriden dominanten Erbgang von $\mathrm{Gr}$ rechnen konnte. Um diese Arbeitshypothese zu prüfen, wurden reziproke Kreuzungen zwischen Tieren des $G r$-Stammes, der nicht reinerbig ist, und

Tabelle 1

Kreuzungen von $\not Q q$ aus dem $G r$-Stamm mit $\hat{o} \hat{o}$ aus dem Normal-Stamm

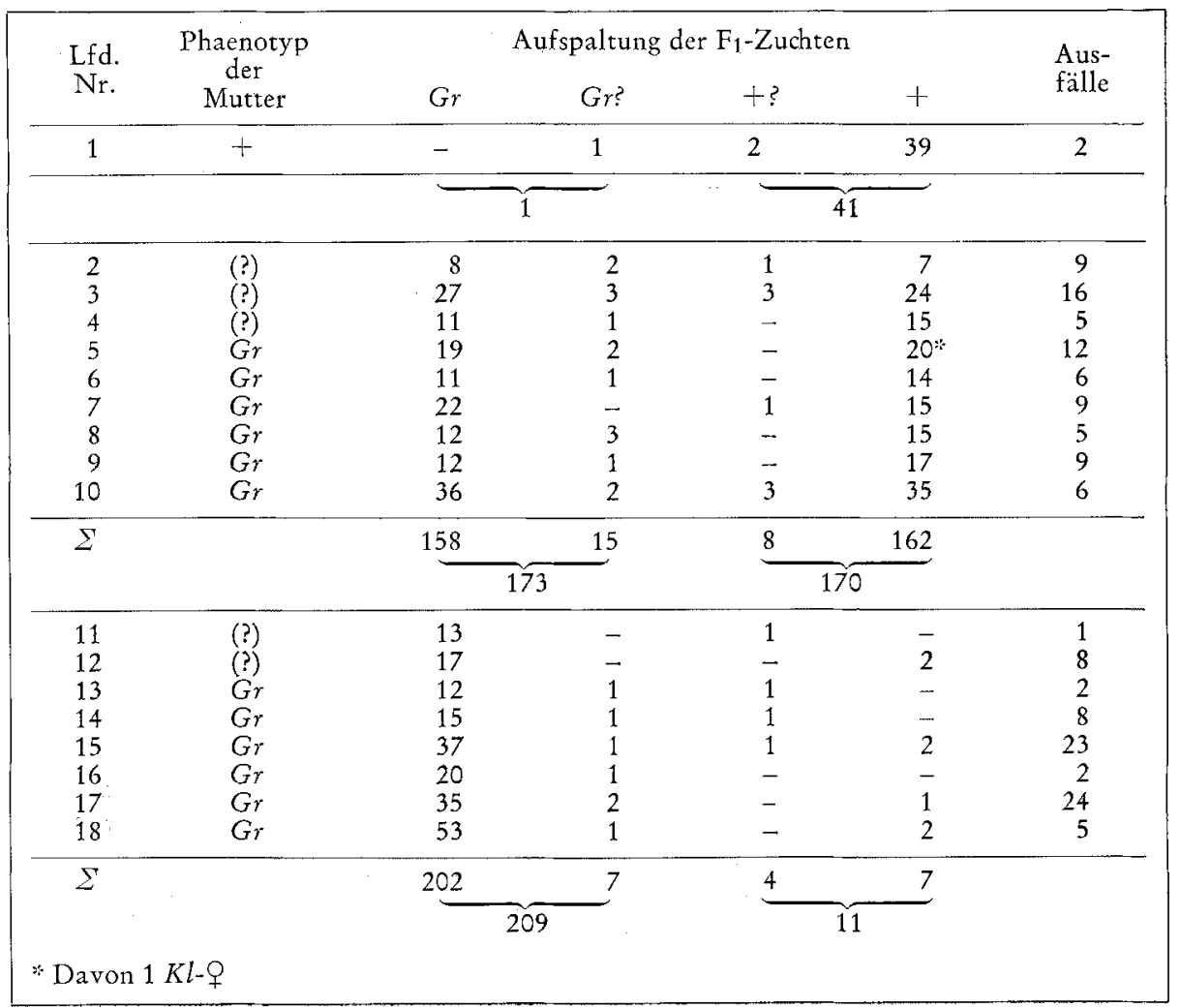


Tieren des Normal-Stammes durchgeführt. Wie aus Tabelle 1 (Kreuzungen: $G r-Q \times$ $+/+-\hat{\delta}$ ) und Tabelle 2 (Kreuzungen: $+/+-q \times G r-\hat{o}$ ) hervorgeht, lassen sich die Nachkommenschaften der beiden reziproken Kreuzungsserien nach ihrem Aufspaltungsergebnis zu je 3 Gruppen ordnen, die den nach der Arbeitshypothese zu erwartenden Kreuzungskombinationen $+1+\times+1+, G r /+\times+1+$ und $G r / G r \times+1+$ entsprechen. Die bei einem Vergleich mit den Erwartungswerten erkennbaren Abweichungen vom normalen Verhalten eines monohybrid dominanten Erbganges machen eine genauere Analyse notwendig.

Die 1. Gruppe der Kreuzung $G r-q \times+/+-\delta$ enthält nur eine Nachkommenschaft, die sich mit einer Ausnahme nur aus +-Tieren zusammensetzt (Tab. 1, Ifd.

Tabelle 2

Kreuzungen von $Q 9+$ aus dem Normal-Stamm mit $\delta \hat{\delta}$ aus dem $G r$-Stamm

\begin{tabular}{|c|c|c|c|c|c|c|}
\hline \multirow{2}{*}{$\begin{array}{l}\text { Lfd. } \\
\text { Nr. }\end{array}$} & \multirow{2}{*}{$\begin{array}{c}\text { Phaenotyp } \\
\text { der } \\
\text { Mutter }\end{array}$} & \multicolumn{4}{|c|}{ Aufspaltung der $F_{1}$-Zuchten } & \multirow{2}{*}{$\begin{array}{l}\text { Aus } \\
\text { fäll }\end{array}$} \\
\hline & & $G r$ & $G r ?$ & $+?$ & + & \\
\hline 1 & + & - & - & 1 & 47 & 5 \\
\hline $2^{*}$ & + & 3 & 1 & 4 & 19 & 1 \\
\hline $3^{*}$ & + & - & - & 3 & 18 & 1 \\
\hline \multirow[t]{2}{*}{$\Sigma$} & & 3 & 1 & 8 & 84 & \\
\hline & & \multicolumn{2}{|c|}{4} & \multicolumn{2}{|c|}{92} & \\
\hline 4 & + & 10 & 1 & - & 19 & 2 \\
\hline 5 & + & 14 & - & 2 & 49 & 7 \\
\hline 6 & + & 15 & - & - & 42 & - \\
\hline 7 & + & 25 & 1 & 1 & 57 & 2 \\
\hline 8 & -1 & 20 & 1 & - & 26 & 4 \\
\hline 9 & + & 14 & 2 & 1 & 27 & 9 \\
\hline 10 & + & 20 & - & - & 17 & 3 \\
\hline 11 & + & 32 & - & - & 26 & $\therefore$ \\
\hline 12 & + & 15 & - & - & 22 & 1 \\
\hline \multirow[t]{2}{*}{$\Sigma$} & & 165 & 5 & 4 & 285 & \\
\hline & & \multicolumn{2}{|c|}{170} & \multicolumn{2}{|c|}{289} & \\
\hline 13 & + & 14 & - & - & - & 3 \\
\hline 14 & + & 21 & - & - & - & - \\
\hline 15 & + & 28 & 1 & - & - & 1 \\
\hline 16 & + & 50 & - & - & - & - \\
\hline 17 & + & 40 & - & - & - & - \\
\hline 18 & + & 36 & - & - & 1 & 5 \\
\hline 19 & + & 49 & - & - & 3 & 1 \\
\hline 20 & + & 57 & 2 & - & - & - \\
\hline 21 & + & 48 & - & - & - & - \\
\hline 22 & + & 48 & - & - & 1 & - \\
\hline 23 & + & 43 & 1 & - & 1 & - \\
\hline 24 & + & 38 & - & - & 1 & - \\
\hline \multirow[t]{2}{*}{$\Sigma$} & & 472 & 4 & $=$ & 7 & \\
\hline & & \multicolumn{2}{|c|}{476} & \multicolumn{2}{|c|}{7} & \\
\hline
\end{tabular}


Nr. 1). Von dem einen bei $G r$ ? eingeordneten Ausnahme-Tier wurde eine $\mathrm{F}_{2}$-Generation ausgewertet. Sie bestand aus $10+$-Tieren und keinem Gr-Tier. Bei dem AusnahmeTier handelt es sich somit nicht um ein $G r /+-q$, für das eine 1:1-Erwartung entsprechend den im folgenden Abschnitt angeführten Ergebnissen gelten würde, sondern genetisch um ein Wildtyp- 9 , das einige ziemlich große Eier legte und daher verdachtsweise als $G r$ ? klassifiziert wurde. Man kann daher diese Gruppe als Ergebnis einer $+/+-X+1+-K r e u z u n g$ im Sinne der Arbeitshypothese betrachten.

Die 2. Gruppe (Tab. 1, Ifd. Nr. 2 bis 10) setzt sich aus 9 Nachkommenschaften mit 1:1-Aufspaltungen zwischen $G r$-Tieren und +-Tieren zusammen. Die Úbereinstimmung mit einem 1:1-Verhältnis ist mit $173 \mathrm{Gr}$ - zu $170+$-Tieren ausgezeichnet, die Homogenität der Gruppe mit $p=0,97$ sehr hoch $\left(\chi^{2}\right.$-Test). Diese Gruppe entspricht der erwarteten Aufspaltung bei Kreuzungen des Typs $G r /+\times+1+$.

Die 3. Gruppe (Tab. 1, lfd. Nr. 11 bis 18) umfaßt 8 Nachkommenschaften, die vollständig oder fast vollständig aus $G r$-Tieren bestehen. Diese Gruppe stimmt noch am ehesten überein mit den zu erwartenden Nachkommenschaften aus $G r / G r-\times$ $+/+$-Paarungen. Die aufgetretenen +- und + ?-Ausnahmetiere machen allerdings zusammen 5,0\% aus und passen schlecht zu dieser Interpretation. Ihr Verhalten wird genauer auf den Seiten 303 bis 306 untersucht.

In der reziproken Kreuzung $+1+-9 \times G \gamma$ - $\partial$ enthält die 1. Gruppe (Tab. 2, Ifd. Nr. 1 bis 3) 3 Nachkommenschaften, die ausschließlich oder fast ausschließlich aus +- Tieren bestehen. Die lfd. Nr. 1 enthält nur +-Tiere, kann also als Ergebnis einer $+1+x+1+-$ Kreuzung angesehen werden.

Die beiden übrigen (lfd. Nr. 2 und 3) machen Schwierigkeiten hinsichtlich der Zuordnung der $ㅇ ㅜ$ zu den verschiedenen Phaenotypen-Klassen. Auch die als + -Tiere klassifizierten 우우 legten etwas größere $\hat{\delta}$-Eier als der Durchschnitt der +-Tiere in anderen Kreuzungen. Einige $F_{1}-9 Q$ der Zucht Nr. 2 waren als $G r$ bzw. Gr? zu bezeichnen. Ihre Nachkommenschaften konnten nicht geprüft werden. Nr. 2 und 3 besitzen aber denselben Vater, so daß die Gr- und Gr?-Tiere vermutlich nicht genetisch verschieden von den restlichen sind. Diese Klassifizierungsschwierigkeiten von sehr vielen intermediären Formen traten in einer anderen Zucht (s. Tab. 3, Ifd. Nr. 1 und Tab. 7, lfd. Nr. 1) bei zwei Nachkommenschaften desselben Muttertieres mit verschiedenen $\delta \delta$ noch einmal auf. Die Erscheinung ist also offenbar erblich.

Die 2. Gruppe (Tab. 2, Ifd. Nr. 4 bis 12) umfaßt 9 Nachkommenschaften mit größeren $G r$ - und +-Anteilen. Diese Gruppe sollte als Ergebnis von $+/+-\times G r /+-$ Kreuzungen angesehen werden. 1:1-Verhältnisse sind jedoch nur in wenigen Nachkommenschaften realisiert, die Mehrzahl zeigt einen Überschuß an +-Tieren. Die Abweichung vom 1:1-Verhältnis ist signifikant $(p \ll 0,001)$. Aber das Material ist nicht homogen $(p \approx 0,001)$, die älteren Zuchten (lfd. Nr. 4 bis 7) weichen deutlich stärker vom 1:1-Verhältnis ab als die jüngeren (Ifd. Nr. 8 bis 12). Die unterschiedlichen $G r-$ Anteile und die Abweichungen vom 1:1-Verhältnis können nicht durch Ausfälle in einer Klasse erklärt werden, wie die folgende Gegenüberstellung von $G r$-Anteilen und Anteilen an Ausfällen ( $q$ + ohne Eiablage) erkennen läßt:

$$
\begin{array}{rrrrrrrrrr}
G r \%: & 21,5 & 26,3 & 31,0 & 36,4 & 36,7 & 40,5 & 44,7 & 54,1 & 55,2 \\
\text { Ausfall \%: } & 9,5 & 0,0 & 2,3 & 17,0 & 6,3 & 2,6 & 7,8 & 7,5 & 0,0
\end{array}
$$


Man muß in Anbetracht der guten 1:1-Aufspaltung in der reziproken Kreuzung den Grund für die Abweichung vom 1:1-Verhältnis in einer Benachteiligung der GrSpermien sehen. Handelt es sich um Certation, so müßte sich eine Verschiebung des $G r$-Anteils von frühen zu späten Eiablagen bemerkbar machen, da ja durch eine größere Befruchtungshäufigkeit der + -Spermien im Laufe der Zeit der Anteil der Gr-Spermien und damit deren Befruchtungschancen steigen müßten. Teilt man zur Prüfung dieser Vorstellung die 4 zahlenmäßig größten Nachkommenschaften der Kreuzung $+1+x$ $G r /+$ nach frühen und späten Ablagen auf und wertet beide Gruppen getrennt aus (Tab. 3), so ergeben sich für beide Gruppen fast die gleichen Werte, nämlich für die frühen Ablagen 33,1\% Gr-Individuen und für die späten Ablagen 32,8\%. Es findet sich also kein Hindernis dafür, daß Certation die Ursache für die Abweichung vom 1:1-Verhältnis ist. Die Zusammenstellung in Tabelle 3 bietet im übrigen auch keinen Hinweis dafür, daß als Ursache für die Heterogenität der Zahlenverhältnisse das evtl. unterschiedliche Alter der Muttertiere verantwortlich sein könnte. Alle 4 Zuchten verhalten sich trotz verschiedener $G r$-Anteile in der ersten und zweiten Hälfte gleich. Möglicherweise ist ein Teil der $G r$-Spermien so verändert, daß sie überhaupt nicht mehr zur Befruchtung befähigt sind. Dieser Anteil müßte Schwankungen unterworfen sein, wie sich aus der Heterogenität des $G r$-Anteils bei diesem Kreuzungstyp ergibt.

Die 3. Gruppe (Tab. 2, Ifd. Nr. 13 bis 24) schließlich setzt sich aus reinen und fast reinen $G r$-Nachkommenschaften zusammen. Sie entspricht in etwa dem erwarteten Ergebnis einer $+1+-\times G r / G r-\mathrm{K}$ reuzung. Ebenso wie in der entsprechenden Gruppe der reziproken Kreuzung sind aber hier unerwartete Wildtyp-Ausnahmetiere zu finden. Sie treten in einer Häufigkeit von insgesamt 1,4\% auf. Das genetische Verhalten der Ausnahme- $q \circ$ wird weiter unten dargestellt.

Tabelle 3

Aufspaltungsverhältnis in frühen und späten Eiablagen bei Kreuzungen mit $G r /+-\hat{\partial} \hat{\delta}$

\begin{tabular}{|c|c|c|c|c|c|c|c|c|}
\hline \multirow{2}{*}{$\begin{array}{l}\text { Lfd. } \\
\text { Nr. }\end{array}$} & \multicolumn{4}{|c|}{ Frühe Ablagen } & \multicolumn{4}{|c|}{ Späte Ablagen } \\
\hline & $G r$ & $G r ?$ & $+?$ & + & $G r$ & Gr? & $+?$ & + \\
\hline 1 & 20 & - & - & 21 & 12 & - & - & 5 \\
\hline 2 & 7 & - & - & 19 & 18 & 1 & 1 & 38 \\
\hline 3 & 9 & - & - & 17 & 6 & - & - & 25 \\
\hline 4 & 6 & - & 1 & 27 & 8 & - & 1 & 22 \\
\hline \multirow[t]{2}{*}{$\Sigma$} & 42 & - & 1 & 84 & 44 & 1 & 2 & 90 \\
\hline & \multicolumn{2}{|c|}{42} & \multicolumn{2}{|c|}{85} & \multicolumn{2}{|c|}{45} & \multicolumn{2}{|c|}{92} \\
\hline$\% / 0$ & \multicolumn{2}{|c|}{33,1} & & & \multicolumn{2}{|c|}{32,8} & & \\
\hline
\end{tabular}

\section{Rückkreuzungen}

Aus der Tatsache, daß $F_{1}-q 9$ aus der Kreuzung von $G r$-Tieren mit Tieren aus dem Normal-Stamm das Merkmal „Gr" zeigen, muß auf einen dominanten Erbgang von $G r$ geschlossen werden. Wegen der auftretenden Differenzen zwischen dem Er- 
Tabelle 4

$\mathrm{F}_{2} \mathrm{R}$ aus $+/+\times G r / G r$ und $G r / G r \times+1+$

\begin{tabular}{|c|c|c|c|c|c|}
\hline \multirow{2}{*}{$\begin{array}{l}\text { Lfd. } \\
\text { Nr. }\end{array}$} & \multirow{2}{*}{$\begin{array}{l}\text { Phaenotyp } \\
\text { der } \\
\text { Mutter }\end{array}$} & \multicolumn{4}{|c|}{ Aufspaltung der $\mathrm{F}_{2} \mathrm{R}$-Zuchten } \\
\hline & & Gr & Gr? & $+?$ & + \\
\hline $1^{*}$ & Gr & 27 & 8 & 8 & 14 \\
\hline 2 & Gr & 43 & - & - & 38 \\
\hline 3 & Gr & 21 & - & - & 30 \\
\hline 4 & Gr & 27 & - & - & 30 \\
\hline 5 & $G r$ & 16 & - & - & 20 \\
\hline 6 & Gr & 10 & - & - & 16 \\
\hline 7 & $G r$ & 12 & 1 & -. & 16 \\
\hline 8 & Gr & 22 & 2 & - & 17 \\
\hline 9 & $G r$ & 17 & - & - & 9 \\
\hline 10 & Gr & 16 & - & - & 13 \\
\hline 11 & Gr & 12 & - & - & 25 \\
\hline 12 & Gr & 17 & - & 1 & 21 \\
\hline 13 & Gr & 11 & - & - & 9 \\
\hline 14 & $G r$ & 21 & - & - & 19 \\
\hline 15 & $G r$ & 15 & - & - & 18 \\
\hline 16 & $G^{r}$ & 24 & - & - & 18 \\
\hline 17 & $G r$ & 17 & - & - & 24 \\
\hline 18 & $G r$ & 17 & - & - & 19 \\
\hline 19 & $G r$ & 34 & 1 & - & 15 \\
\hline 20 & $G r$ & 31 & - & - & 29 \\
\hline 21 & $G r$ & 14 & - & - & 8 \\
\hline 22 & $G r$ & 15 & - & - & 11 \\
\hline 23 & $G_{r}^{r}$ & 10 & - & - & 12 \\
\hline 24 & $G r$ & 43 & - & 1 & 33 \\
\hline 25 & $G r$ & 33 & 2 & - & 17 \\
\hline 26 & $G r$ & 65 & 3 & -. & 56 \\
\hline 27 & $G r$ & 50 & - & - & 63 \\
\hline 28 & $G r$ & 24 & - & - & 26 \\
\hline \multirow[t]{2}{*}{$\Sigma$} & & 664 & 17 & 10 & 626 \\
\hline & & \multicolumn{2}{|c|}{681} & \multicolumn{2}{|c|}{636} \\
\hline
\end{tabular}

gebnis und der Erwartung muß aber geprüft werden, ob der Erbgang tatsächlich, wie angenommen, monohybrid ist. Zur Beantwortung dieser Frage wurde eine größere Zahl von $\mathrm{F}_{2} \mathrm{R}$-Zuchten ausgewertet. $G r$ - +9 aus einheitlichen oder fast einheitlichen $G r$-Nachkommenschaften, die also nach der vorläufigen Interpretation aus $G r / G r-X$ $+/+-$ bzw. $+1+-\times G r / G r$-Kreuzungen resultieren, wurden mit $\hat{\delta} \hat{\delta}$ aus dem Normal-Stamm rückgekreuzt. Aus der Kreuzung $+/+\times G r / G r$ wurden $24 G r$ - + , , aus der reziproken Kreuzung $4 \mathrm{Gr}$ - $9+9$ mit $+1+-\delta \hat{\delta}$ angepaart. Die geprüften $\mathrm{F}_{2} \mathrm{R}-\mathrm{Nach}-$ kommenschaften (Tab. 4) enthielten insgesamt 51,7\% Gr-Tiere, die Übereinstimmung mit dem 1:1-Verhältnis ist ausreichend $(0,2<p<0,3)$, die Homogenität ebenfalls $(p \approx 0,1)$, obwohl einzelne extreme Abweichungen, wie z. B. 35:15 (lfd. Nr. 19) zu verzeichnen waren.

Dihybride Erbgänge und solche mit Beteiligung von mehr Faktoren hätten bei der erwiesenen Dominanz des Erbganges in der $\mathrm{F}_{2} \mathrm{R}$ sowohl bei komplementärer wie auch bei additiver Wirkung $G r$-Anteile von $1 / 4$ und weniger (bei di-, tri-, tetra-hybriden 
Tabelle 5

$\mathrm{F}_{3} \mathrm{R}$ aus $+/+\times G r / G r$

\begin{tabular}{|c|c|c|c|c|c|}
\hline \multirow{2}{*}{$\begin{array}{l}\text { Lfd. } \\
\text { Nr. }\end{array}$} & \multirow{2}{*}{$\begin{array}{c}\text { Phaenotyp } \\
\text { der } \\
\text { Mutter }\end{array}$} & \multicolumn{4}{|c|}{ Aufspaltung der $F_{3} R-$ Zuchten } \\
\hline & & Gr & Gr? & $+?$ & \\
\hline 1 & + & - & - & - & 32 \\
\hline 2 & + & - & - & - & 14 \\
\hline 3 & + & - & - & - & 20 \\
\hline \multirow[t]{2}{*}{$\Sigma$} & & - & $=$ & - & 66 \\
\hline & & \multicolumn{2}{|c|}{-} & \multicolumn{2}{|c|}{66} \\
\hline 4 & Gr & 18 & - & - & 29 \\
\hline 5 & Gr & 14 & - & - & 12 \\
\hline \multirow[t]{2}{*}{$\Sigma$} & & 32 & $=$ & - & 41 \\
\hline & & \multicolumn{2}{|c|}{32} & \multicolumn{2}{|c|}{41} \\
\hline
\end{tabular}

usw. Erbgängen) erwarten lassen. Das Ergebnis spricht demnach eindeutig für einen monohybriden dominanten Erbgang.

Auch probeweise durchgeführte weitere Rückkreuzungen der $\mathrm{F}_{2} \mathrm{R}-\phi+9+$ mit $+/+-\delta$ aus dem Normal-Stamm verhielten sich erwartungsgemäß (Tab. 5): $q$ 우 mit +Phaenotyp ergaben nur +-Nachkommen, Kreuzungen von $\$+q$ mit $G r$-Phaenotyp spalteten auf im 1:1-Verhältnis.

\section{$F_{2}-Z u c h t e n$ und Prüfung der + -Ausnahmetiere}

Auf Grund der im vorigen Abschnitt dargestellten Ergebnisse der Rückkreuzungen muß man die vorläufige Deutung als richtig ansehen. Um so auffälliger ist das Auftreten der 5,0\% +-Ausnahme- +9 in den Nachkommenschaften von $\mathrm{Gr} / \mathrm{Gr}-\mathrm{X}+\mathrm{H}+$ Kreuzungen und den 1,4\% +-Ausnahme- 9 \% in der reziproken Kreuzungskombination $+1+\times G r / G r$. Zur Prüfung des Genotyps der Ausnahmen wurden $\mathrm{F}_{2}$ Zuchten aus der $G r / G r-\times+/+-$ Kreuzung angelegt, und zwar sowohl von $G r-q 9$ (Tab. 6, lfd. Nr. 1 bis 6) als auch von + -Ausnahme- 9 (T) (Tab. 6, Ifd. Nr. 7 bis 11).

Die $\mathrm{F}_{2}$-Zuchten von $G r$ - +9 ergaben $167 \mathrm{Gr}$-Nachkommen und 99 +- Nachkommen und damit einen Gr-Anteil von $62,8 \%$ in den Nachkommenschaften. Dic Abweichung vom 3:1-Verhältnis mußte wegen der Benachteiligung der Gr-Spermien im Vergleich zu den + -Spermien erwartet werden. Auf Grund der Ergebnisse der $+/+-\times G r /+-K r e u z u n g e n$ (Tab. 2, Ifd. Nr. 4 bis 12) läßt sich ein durchschnittlicher $\mathrm{Gr}$-Anteil von 68,5\% Gr-Tieren in der $\mathrm{F}_{2}$ erwarten bei Annahme einer ungeminderten Vitalität der $G r / G r$-Nachkommen. Der gefundene Wert von 62,8\% bedeutet eine gute Übereinstimmung mit dem Erwartungswert in Anbetracht der möglichen Fehler (starke Schwankungen bei Kreuzungen mit $G r /+-\hat{\partial} \hat{\delta}$, mögliche Beteiligung von $+/+$-Ausnahme- $\delta$ ô. .

Von den +-Ausnahme- $+q$ wurden $5 \mathrm{~F}_{2}$-Zuchten angesetzt (Tab. 6, lfd. Nr. 7 
bis 11). Alle 5 Zuchten spalteten auf. Bei einer Zucht (lfd. Nr. 9) mit allerdings geringer Nachkommenzahl überwogen die $G r$-Tiere, in den übrigen 4 waren die +-Tiere zahlenmäßig meist deutlich überlegen. Der Anteil der $G r$-Tiere betrug insgesamt 42,5\%. Er entspricht damit dem Ergebnis einer Kreuzung des Typs $+/+\times G r /+(v g l$. Tab. 2, lfd. Nr. 4 bis 12). Man kann also damit rechnen, daß es sich bei der Mehrzahl der + -Ausnahmetiere genetisch um $+/+-$ Tiere handelt.

Tabelle 6

$F_{2}$ aus $G r / G r \times+1+$, lfd. $\mathrm{Nr} .7$ bis $11+$-Ausnahme- 99

\begin{tabular}{|c|c|c|c|c|c|}
\hline \multirow{2}{*}{$\begin{array}{l}\text { Ifd. } \\
\text { Nr. }\end{array}$} & \multirow{2}{*}{$\begin{array}{c}\text { Phaenotyp } \\
\text { der } \\
\text { Mutter }\end{array}$} & \multicolumn{4}{|c|}{ Aufspaltung der Zuchten } \\
\hline & & $G r$ & Gr? & $+?$ & + \\
\hline 1 & $G r$ & 29 & 1 & - & 27 \\
\hline 2 & $G r$ & 22 & - & 1 & 9 \\
\hline$\overline{3}$ & $G r$ & 17 & - & 1 & 8 \\
\hline 4 & Gr & 45 & 1 & - & 27 \\
\hline 5 & $G r$ & 32 & 2 & - & 16 \\
\hline 6 & Gr & 18 & - & - & 10 \\
\hline \multirow[t]{2}{*}{$\Sigma$} & & 163 & 4 & $\underbrace{2}$ & 97 \\
\hline & & \multicolumn{2}{|c|}{167} & \multicolumn{2}{|c|}{99} \\
\hline 7 & + & 13 & - & - & 22 \\
\hline 8 & + & 21 & - & - & 25 \\
\hline 9 & + & 9 & - & 1 & 4 \\
\hline 10 & + & 20 & - & - & 34 \\
\hline 11 & + & 14 & - & 1 & 17 \\
\hline \multirow[t]{2}{*}{$\Sigma$} & & 77 & - & 2 & 102 \\
\hline & & \multicolumn{2}{|c|}{77} & \multicolumn{2}{|c|}{104} \\
\hline
\end{tabular}

$\mathrm{Zu}$ einem sicheren Nachweis dafür, daß die +-Ausnahme- 90 auch genetisch Wildtypcharakter besitzen, ist eine Anpaarung mit $+1+-\hat{\delta} \hat{\delta}$ notwendig. Der Phaenotyp ist aber nur bei schon begatteten $O+$, die Eier ablegen, erkennbar. Da normalerweise die erste Kopulation mit einem Kokongeschwister- $\delta$ gleich beim Schlüpfen aus dem Kokon erfolgt, und man wegen der Seltenheit der Ausnahmen nicht alle weiblichen Nachkommen isoliert anpaaren kann, muß man abwarten, bis der Spermienvorrat von der ersten Kopulation aufgebraucht ist und die $ㅇ ㅜ$ dann gezielt mit einem $+1+-\hat{b}$ anpaaren.

Um diese Methode zu testen, wurden 5 Mütter der $+/+-\times G r / G r$-Kreuzung (Tab. 2, lfd. Nr. 19 bis 23), nachdem ihre Spermien verbraucht waren, ein zweites Mal angepaart, diesmal mit $\hat{O} \hat{\delta}$ aus dem Normal-Stamm (Tab. 7). Die Zusammensetzung der Nachkommenschaft änderte sich nach der zweiten Anpaarung schlagartig. Während die Kreuzung mit den $G r$ - $\partial \hat{\partial}$ neben $5+$-Tieren $248 G r$-Tiere erbrachte, waren in der ersten Ablage nach der neuen Anpaarung neben $62+$-Tieren nur noch $3 \mathrm{Gr}$-Tiere zu finden, und in den folgenden Ablagen traten überhaupt keine $G r$-Tiere mehr auf (83 +-Tiere, kein $G r$-Tier). Unter den bald nach der neuen Anpaarung abgelegten Eiern waren offenbar noch einzelne von alten Restspermien befruchtet, wäh- 
Tabelle 7

$+/+-\phi Q$ zweimal angepaart, 1. Paarung mit $G r / G r-\hat{\jmath}, 2$. Paarung mit $+/+-\hat{\jmath}$

\begin{tabular}{|c|c|c|c|c|c|c|c|c|c|c|c|c|c|}
\hline \multirow{2}{*}{$\begin{array}{l}\text { Lfd. } \\
\text { Nr. }\end{array}$} & \multirow{2}{*}{$\begin{array}{c}\text { Phaenotyp } \\
\text { der } \\
\text { Mutter }\end{array}$} & \multirow{2}{*}{\multicolumn{4}{|c|}{$\begin{array}{l}\text { Nachkommen aus der } \\
\text { 1. Paarung }\end{array}$}} & \multicolumn{8}{|c|}{ Nachkommen aus der 2. Paarung } \\
\hline & & & & & & $G r$ & $\begin{array}{l}\text { 1. A } \\
\text { Gr? }\end{array}$ & $\begin{array}{c}\text { blage } \\
+ \text { ? }\end{array}$ & + & $G r^{\mathrm{w}}$ & $\begin{array}{c}\text { eitere } \\
\text { Gr? }\end{array}$ & $\begin{array}{c}\text { Ablag } \\
+?\end{array}$ & ${ }^{e n}+$ \\
\hline 1 & + & 49 & - & - & 3 & \multicolumn{4}{|c|}{ nicht isoliert } & - & - & - & 10 \\
\hline 2 & + & 57 & 2 & - & - & \multicolumn{4}{|c|}{ nicht isoliert } & - & - & - & 37 \\
\hline 3 & + & 48 & - & - & - & 1 & - & - & 21 & - & - & - & 21 \\
\hline 4 & + & 48 & - & - & 1 & 2 & - & - & 22 & - & - & - & 5 \\
\hline 5 & + & 43 & 1 & - & 1 & - & - & - & 19 & - & - & - & 10 \\
\hline \multirow[t]{2}{*}{$\Sigma$} & & 245 & 3 & - & 5 & 3 & $=$ & - & 62 & - & 二 & $=$ & 83 \\
\hline & & \multicolumn{2}{|c|}{248} & \multicolumn{2}{|c|}{3} & \multicolumn{2}{|l|}{3} & \multicolumn{2}{|c|}{62} & \multicolumn{2}{|c|}{-} & \multicolumn{2}{|c|}{83} \\
\hline
\end{tabular}

Tabelle 8

$(+/+\times G r / G r) F_{1}-$ 오 zweimal angepaart; 1. Paarung mit Geschwister- $\hat{\partial} \hat{\delta}$, 2. Paarung mit $+1+-\delta^{+} \delta$. Lfd. Nr. 5 bis $8:+$-Ausnahme- +9 unter $G r$-Geschwistern

\begin{tabular}{|c|c|c|c|c|c|c|c|c|c|c|c|c|c|}
\hline \multirow{2}{*}{$\begin{array}{l}\text { Lfd. } \\
\text { Nr. }\end{array}$} & \multirow{2}{*}{$\begin{array}{c}\text { Phaenotyp } \\
\text { der } \\
\text { Mutter }\end{array}$} & \multicolumn{4}{|c|}{$\begin{array}{c}\text { Nachkommen aus der } \\
\text { 1. Paarung }\end{array}$} & \multicolumn{8}{|c|}{ Nachkommen aus der 2. Paarung } \\
\hline & & $G r$ & $\begin{array}{l}\text { 1. P } \\
\text { Gr? }\end{array}$ & $\begin{array}{c}\operatorname{arung} \\
+?\end{array}$ & + & $G r$ & 1. A & $\begin{array}{c}\text { blage } \\
+?\end{array}$ & + & $G r$ & $\begin{array}{c}\text { veitere } \\
\text { Gr? }\end{array}$ & $\begin{array}{c}\text { Ablag } \\
+?\end{array}$ & + \\
\hline $1 \%$ & $G r$ & 34 & 11 & 5 & 7 & 12 & 1 & 3 & 5 & 27 & 8 & 8 & 14 \\
\hline 2 & Gr & 1 & - & - & 23 & 7 & - & - & 11 & 43 & - & - & 38 \\
\hline 3 & $G r$ & 35 & - & - & 23 & 2 & - & - & 6 & 21 & - & - & 30 \\
\hline 4 & $G r$ & 32 & - & - & 20 & 13 & - & - & 11 & 27 & -- & - & 30 \\
\hline \multirow[t]{2}{*}{$\Sigma$} & & 115 & 11 & 5 & 73 & 34 & 1 & 3 & 33 & 118 & 8 & 8 & 112 \\
\hline & & \multicolumn{2}{|c|}{126} & \multicolumn{2}{|c|}{78} & \multicolumn{2}{|c|}{35} & \multicolumn{2}{|c|}{36} & 12 & & \multicolumn{2}{|c|}{120} \\
\hline 5 & + & 14 & 1 & 3 & 68 & 1 & 1 & - & 1 & $1^{*}$ & - & - & 59 \\
\hline 6 & + & 10 & - & 1 & 35 & - & - & - & - & - & - & - & - \\
\hline 7 & + & 5 & - & - & 37 & - & - & - & - & - & - & - & 8 \\
\hline 8 & + & 12 & 2 & 1 & 34 & - & - & - & 25 & - & - & - & 11 \\
\hline \multirow[t]{2}{*}{$\Sigma$} & & 41 & 3 & 5 & 169 & 1 & 1 & - & 26 & 1 & - & - & 78 \\
\hline & & 44 & & & 74 & & & & 6 & 1 & & & 78 \\
\hline
\end{tabular}

rend in den folgenden Ablagen alle Eier von den Spermien des zweiten $\hat{o}$ befruchtet worden waren. Die Methode eignet sich also, Kreuzungen desselben $q$ mit zwei genetisch verschiedenen $\hat{\delta} \hat{\delta}$ durchzuführen.

Von den Nachkommen der Kreuzung $+1+\times G r / G r$ (Tab. 2, 3. Gruppe) wurden auf diese Weise $4 \mathrm{Gr}$ - $q+$ und $4+$-Ausnahme- +9 getestet. Die erste Paarung war dabei in allen Fällen eine Bruder-Schwester-Paarung, entsprach also wahrscheinlich einer Kreuzung mit einem $G r /+-\hat{O}$, die zweite Kreuzung erfolgte mit einem $+1+-\delta$.

Für die Aufspaltung in den Nachkommenschaften der 4 Gr- 90 (Tab. 8, lfd. Nr. 
1 bis 4, 1. Spalte) gilt dieselbe Erwartung wie für die $F_{2}$ der reziproken Kreuzung (vgl. p. 303). Der gefundene Wert von insgesamt 61,8\% $\mathrm{Gr}$-Tiere liegt sehr nahe bei dem der reziproken Serie $(62,8 \%$ ). Beide stimmen mit der Erwartung $(68,5 \% \mathrm{Gr}$ Tiere, s. o.) überein. Die $\$+q$ wurden nach Ablage der letzten befruchteten Eier mir einem $+1+-\hat{\delta}$ angepaart, so daß von denselben $\phi+q$ außer der $F_{2}$ auch eine $F_{2} R$ erhalten wurde (Tab. 8, Ifd. Nr. 1 bis 4, 2. und 3. Spalte). Diese stimmt mit 49,3\% Gr-Individuen in der ersten neuen Ablage und 51,2\% in den weiteren Ablagen gut mit dem theoretischen Wert von $50,0 \%$ überein.

Von den $4+$-Ausnahme- 99 wurden $4 \mathrm{~F}_{\mathrm{q}}$-Nachkommenschaften erzeugt (Tab. 8, Ifd. Nr. 5 bis 8, 1. Spalte). Alle zeigten ein starkes Uberwiegen der +-Tiere. Der Gesamtanteil der $G r$-Tiere liegt bei 20,3\%. Man kann schon hieraus vermuten, daß es sich bei den Ausnahmetieren genetisch un $+1+$-Tiere handelt. 3 Ausnahmetiere konnten, nachdem ihr Spermienvorrat erschöptt war, in der zweiten Anpaarung mit ô $\delta$ aus dem Normal-Stamm gekreuzt werden. Das Ergebnis (Tab. 8, lfd. Nr. 5 bis 8, 2. und 3. Spalte) ist eindeutig: $104+$-Tieren stehen nur $3 \mathrm{Gr}$-Tiere gegenüber, die sich, wie der Vergleich mit Tabelle 7 zeigt, auf einzelne, noch von der ersten Begattung übriggebliebene Spermien zurückführen lassen. Diese Befunde können nur als Ergebnis einer $+/+-X+1+-K r e u z u n g$ aufgefaßt werden. Die Ausnahme- $q \uparrow$ zeigen also nicht nur phaenotypisch den Wildtyp-Charakter, sondern entsprechen auch genetisch dem Wildtyp.

\section{Spermienanomalien}

Bei der Betrachtung der $+1+-\times G r /+-$ Kreuzungsergebnisse wurde eine Benachteiligung der $G r$-Spermien erschlossen, die anscheinend nicht auf Certation beruht. Eine Untersuchung der lebenden Spermien von $G r$-Tieren bei Phasenkontrast ergab, daß bei einem wechselnden Anteil von ihnen Anomalien auftreten (Abb. 2 und Tab. 9 Nr. 1 bis 15). Manche Spermien besitzen 2 Geißeln, hin und wieder wurden auch Spermien mit 4 Geißeln beobachtet. Bei einer Untersuchung der Spermatiden von $G r-\delta \hat{~} \delta$ wurden solche mit 2 und auch mit 4 Kernen festgestellt. Vermutlich sind also die doppelschwänzigen Spermien nicht durch ein sekundäres Verkleben schon getrennter Elemente, sondern durch ein Nicht-Trennen der Abkömmlinge aus den Reifeteilungen zustande gekommen. In normalen $\hat{o} \hat{o}$ des A III-(Normal-)Stammes konnten Doppelspermien bisher nicht nachgewiesen werden (Abb. 1 und Tab. 9, Ifd. Nr. 16 bis 20). Es bestand daher die Möglichkeit, daß diese morphologischen Anomalien der Spermien in Zusammenhang stehen mit der verminderten Befruchtungshäufigkeit der $G r$-Spermien in der Kreuzung.

Zunächst war zu klären, ob die Anomalien durch die veränderte Ausgangsgröße der $\hat{\delta}$-Eier im $G r$-Stamm verursacht werden. Dazu wurden die Spermien von $\delta \hat{\delta}$ untersucht, deren Größe normal war, da sie von $+1+-q 9$ abstammten, deren Genotyp aber $G r /+$ war als Folge einer Kreuzung $+1+\times G r / G r$. Diese $\delta \hat{\delta}$ enthielten ebenfalls Doppelspermien in einer Häufigkeit von 3,5 bis 7,6\% (Tab. 9, 1fd. Nr. 11 bis 13). Hier ist also der Genotyp des $\hat{\theta}$ für die Spermienanomalien verantwortlich, denn normal große ô $\hat{\mathrm{m}} \mathrm{mit}+1+-$ Genotyp zeigten diese Anomalien nicht (lfd. Nr. 16 bis 20). 
Gleichzeitig scheint aber auch die Größe der Eier, aus denen die $\hat{\partial} \hat{\delta}$ hervorgehen, für derartige Spermienanomalien verantwortlich sein zu können. Die aus normal großen Q-Eiern unter bestimmten Umständen entstehenden atypischen $\delta \hat{\delta}$ enthalten nämlich

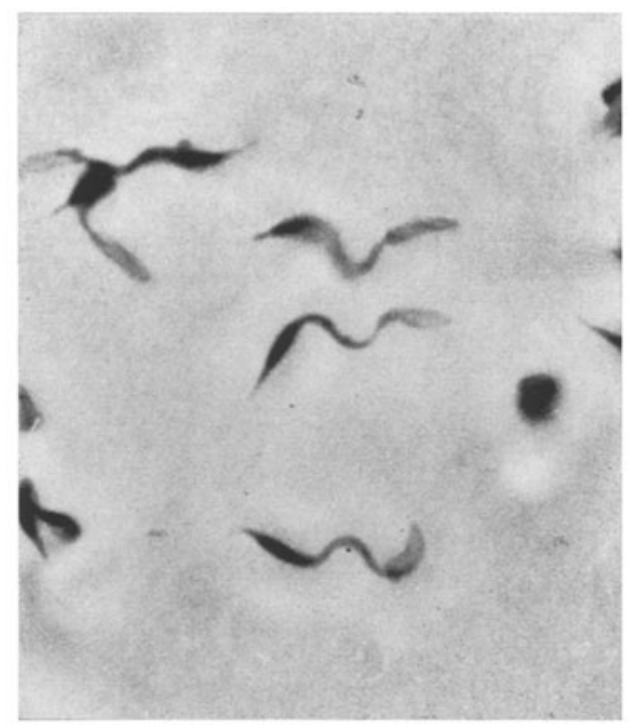

Abb. 1: Lebende Spermien eines $+1+-\hat{\partial}$ von Dinopbilus gyrociliatus, die dunkle Region zeigt die Lage des Kerns an, das bläschenförmige Gebilde liegt am Ende der Geißel. (Phasenkontrast, $1600: 1)$

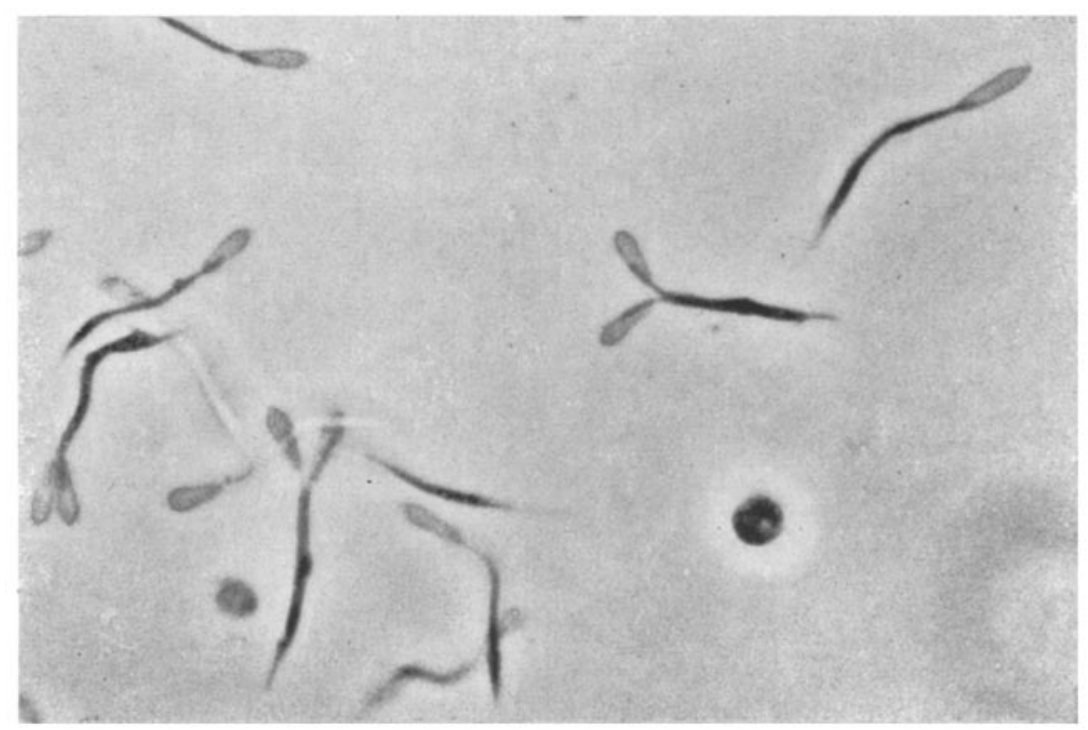

Abb. 2: Lebende Spermien eines $G r /+$ - oder $G r / G r-\hat{\jmath}$, einige Spermien mit doppelter Geißel. (Phasenkontrast, 1600:1) 
auch Doppelspermien und solche mit 4 Geißeln und 4 Kernen. Offenbar kann diese Anomalie durch verschiedene Einflüsse ausgelöst werden.

Für die vermutete Wirkung auf die Befruchtungshäufigkeit der Gr-Spermien könnte man erwarten, daß der Anteil der Doppelspermien in $G r / G r-\hat{\phi} \hat{\delta}$ höher ist als

Tabelle 9

Häufigkeit der Spermien-Anomalien bei $G r / G r-, G r /+-$ und $+1+-\hat{\delta} \hat{\delta}$

\begin{tabular}{|c|c|c|c|c|c|c|}
\hline \multirow{2}{*}{$\begin{array}{l}\text { Lfd. } \\
\mathrm{Nr} \text {. }\end{array}$} & \multirow{2}{*}{$\underset{\substack{\text { Größe des } \\
\hat{\partial}}}{ }$} & \multirow{2}{*}{$\begin{array}{c}\text { Genotyp } \\
\text { des } \delta\end{array}$} & \multicolumn{3}{|c|}{ Spermien mit } & \multirow{2}{*}{$\begin{array}{c}\text { Anteil der } \\
\text { Spermien } \\
\text { mit } \\
2 \text { Geißeln } \\
\text { in } \%\end{array}$} \\
\hline & & & 1 Geißel & 2 Geißeln & 4 Geißeln & \\
\hline $\begin{array}{l}1 \\
2 \\
3\end{array}$ & $\begin{array}{l}\sum_{\text {normal }} \\
>_{\text {normal }}\end{array}$ & $\begin{array}{l}G r / G r \\
G r / G r \\
G r / G r\end{array}$ & $\begin{array}{r}85 \\
79 \\
120\end{array}$ & $\begin{array}{l}5 \\
1 \\
5\end{array}$ & $\begin{array}{l}- \\
\overline{-}\end{array}$ & $\begin{array}{l}5,6 \\
1,3 \\
4,0\end{array}$ \\
\hline $\begin{array}{l}4 \\
5 \\
6 \\
7\end{array}$ & $\begin{array}{l}>\text { normal } \\
\sum_{\text {normal }} \\
>_{\text {normal }} \\
\text { normal }\end{array}$ & $\begin{array}{l}G r / G r \text { oder } G r /+ \\
G r / G r \text { oder } G r /+ \\
G r / G r \text { oder } G r /+ \\
G r / G r \text { oder } G r l+\end{array}$ & $\begin{array}{r}125 \\
32 \\
43 \\
46\end{array}$ & $\begin{array}{r}40 \\
6 \\
8 \\
5\end{array}$ & $\begin{array}{l}2 \\
- \\
- \\
-\end{array}$ & $\begin{array}{r}24,0 \\
15,8 \\
15,7 \\
9,8\end{array}$ \\
\hline $\begin{array}{r}8 \\
9 \\
10\end{array}$ & $\begin{array}{l}>_{\text {normal }} \\
>_{\text {normal }}\end{array}$ & $\begin{array}{l}G r l+ \\
G r l+ \\
G r l+\end{array}$ & $\begin{array}{r}133 \\
59 \\
363\end{array}$ & $\begin{array}{r}16 \\
3 \\
22\end{array}$ & $\begin{array}{l}- \\
- \\
-\end{array}$ & $\begin{array}{r}10,7 \\
4,8 \\
5,7\end{array}$ \\
\hline $\begin{array}{l}11 \\
12 \\
13\end{array}$ & $\begin{array}{l}\text { normal } \\
\text { normal } \\
\text { normal }\end{array}$ & $\begin{array}{l}G r l+ \\
G r l+ \\
G r l+\end{array}$ & $\begin{array}{r}64 \\
110 \\
278\end{array}$ & $\begin{array}{r}3 \\
9 \\
10\end{array}$ & $\begin{array}{l}- \\
- \\
-\end{array}$ & $\begin{array}{l}4,5 \\
7,6 \\
3,5\end{array}$ \\
\hline $\begin{array}{l}14 \\
15\end{array}$ & $\begin{array}{l}>_{\text {normal }} \\
\text { normal }\end{array}$ & $\begin{array}{l}G r /+ \text { oder }+/+ \\
G r /+ \text { oder }+/+\end{array}$ & $\begin{array}{l}77 \\
200 *\end{array}$ & $\begin{array}{l}5 \\
-\end{array}$ & $\begin{array}{l}- \\
-\end{array}$ & 6,1 \\
\hline $\begin{array}{l}16 \\
17 \\
18 \\
19 \\
20\end{array}$ & $\begin{array}{l}\text { normal } \\
\text { normal } \\
\text { normal } \\
\text { normal } \\
\text { normal }\end{array}$ & $\begin{array}{l}+1+ \\
+1+ \\
+1+ \\
+1+ \\
+1+\end{array}$ & $\begin{array}{r}300 \\
84 \\
250 \\
39 \\
42\end{array}$ & $\begin{array}{l}- \\
- \\
- \\
- \\
-\end{array}$ & $\begin{array}{l}- \\
- \\
- \\
- \\
-\end{array}$ & $\begin{array}{l}- \\
\bar{z} \\
\overline{-} \\
-\end{array}$ \\
\hline
\end{tabular}

in $G r /+-\hat{o} \partial$. Diese Erwartung konnte mit den für die Kreuzung $+1+\times G r$ verwendeten $\delta$ o geprüt werden (Tab. 9, 1, 1fd. Nr. 1 bis 3 sowie 8 bis 10). Der erwartete Unterschied ist dabei nicht festzustellen. Die $G r /+-\hat{o} \hat{o}$ (lfd. Nr. 8 bis 10) hatten meist sogar noch etwas mehr Doppelspermien als die $G r / G r-\delta \hat{~} \hat{\partial}$ (lfd. Nr. 1 bis 3).

Es ließ sich demnach zwar nachweisen, daß der Genotyp des $\hat{\delta}$ für die Spermienanomalien verantwortlich ist. Eine Bestätigung dafür, daß die dargestellten Spermienanomalien ursächlich mit der Verschiebung des Spaltungsverhältnisses in $+/+-\times G r /+-$ Kreuzungen zugunsten der $+/+-$ Tiere zusammenhängen, konnte dagegen nicht erbracht werden. Allerdings ließ sich bei dieser Prüfung das eventuell unterschiedliche Alter und sein möglicher den genetischen Anteil überlagernder Einfluß auf die Spermienzusammensetzung nicht prüfen. Falls es sich bei $G r$ um eine Chromosomenmutation handelt, ist die Frage durch Auszählen der abnormen Spermien nicht zu klären, da die Meiosis in homozygoten $\hat{\delta} \hat{\delta}$ möglicherweise weniger gestört ist als in heterozygoten $\hat{b} \hat{\delta}$. 


\section{$K l$-Mutante („Kleinere $\circlearrowleft^{\prime}$-Eier“)}

$K l$-Mutanten traten mehrfach unabhängig voneinander im $G r$-Stamm oder in Kreuzungen mit $G r$ auf. In den Wildtyp-Stämmen wurde $K l$ bisher nicht gefunden. In zwei Fällen gelang es, von den aufgetretenen Mutanten Zuchtstämme $\left(K l^{1}\right.$ und $\left.K l^{3}\right)$ zu isolieren.

Bei den $K l$ - $q$ z zeigten sich Störungen in der Fertilität und ziemlich zahlreiche Entwicklungsstörungen bei den abgelegten Eiern. $K l$ - $Q$ T reagierten außerdem sehr empfindlich auf ungünstige Außenbedingungen, wie Erhöhung der Salzkonzentration usw. Schon bei geringer Nachlässigkeit in der Kontrolle der Zuchtbedingungen legten viele $K l$ - $q$ 우 keine Eier mehr ab.

Tabelle 10

Kreuzungen von $q q$ des $K l^{1}$-Stammes mit $\hat{\delta} \hat{\delta}$ des Normal-Stammes

\begin{tabular}{|c|c|c|c|c|c|c|}
\hline \multirow{2}{*}{$\begin{array}{l}\text { Lff. } \\
\text { Nr. }\end{array}$} & \multirow{2}{*}{$\begin{array}{c}\text { Phaenotyp } \\
\text { der } \\
\text { Mutter }\end{array}$} & \multicolumn{4}{|c|}{ Aufspaltung der Zuchten } & \multirow{2}{*}{$\begin{array}{l}\text { Aus- } \\
\text { fälle }\end{array}$} \\
\hline & & + & $+?$ & Kl? & $K l$ & \\
\hline 1 & + & 50 & - & - & - & 1 \\
\hline 2 & + & 39 & - & - & - & - \\
\hline \multirow[t]{2}{*}{$\Sigma$} & & 89 & - & - & - & \\
\hline & & \multicolumn{2}{|c|}{89} & \multicolumn{2}{|c|}{-} & \\
\hline 3 & $K l$ & 4 & - & - & - & 4 \\
\hline 4 & $K l$ & 4 & - & - & 2 & 2 \\
\hline 5 & $K l$ & 3 & - & - & $\overline{-}$ & 6 \\
\hline 6 & $K l$ & 1 & 1 & - & 2 & 4 \\
\hline 7 & $K l$ & 21 & 1 & 3 & 12 & 14 \\
\hline \multirow[t]{2}{*}{$\Sigma$} & & 33 & 2 & 3 & 16 & \\
\hline & & & & & & \\
\hline
\end{tabular}

Es gelang nicht, die beiden $K l$-Stämme reinerbig zu züchten. Isolierte Nachkommen von $K l$ - $q \circ$ spalteten immer wieder auf in Wildtyp-Individuen und $K l$-Tiere, wobei der $K l$-Anteil selten $1 / 2$ erreichte oder überschritt und niemals über $2 / 3$ hinausging. Reine $K l$-Nachkommenschaften wurden nie erhalten. Die herausspaltenden $+-q Q$ brachten in der Nachzucht immer wieder nur +-Nachkommen hervor. Diese Daten machen die Annahme wahrscheinlich, daß $K l$ einen dominanten, vermutlich monohybriden Erbgang besitzt, und daß entweder homozygote Individuen nicht lebensfähig oder jedenfalls nicht fertil sind und bzw. oder $K V+-\hat{\delta} \hat{\delta}$ im allgemeinen nicht zur Begattung kommen oder ebenfalls nicht fertil sind.

Für die Kreuzungen wurden $q q$ aus den beiden $K l$-Stämmen und $\delta \hat{\delta}$ aus dem Normal-Stamm benutzt. Da die $K l$-Stämme nicht reinerbig zu züchten waren, traten unter den angepaarten $\$ Q q$ beide nach ihren abgelegten Eiern unterscheidbaren $ᄋ$-Klassen auf: phaenotypisch normale $q q(+-q q)$ und $q q$, die kleinere $\delta$-Eier legten $(K l$ ૧q).

Die Kreuzung von $+-9 q$ des $K l^{1}$-Stammes mit $\hat{o} \hat{o}$ aus dem Normal-Stamm ergab in 2 Zuchten nur $+-N a c h k o m m e n(T a b .10,1 f d . N r .1$ und 2) kann also als $+1+-X$ 
Tabelle 11

Kreuzungen von $q Q$ des $K l^{3}$-Stammes mit $\hat{\partial} \hat{o}$ des Normal-Stammes

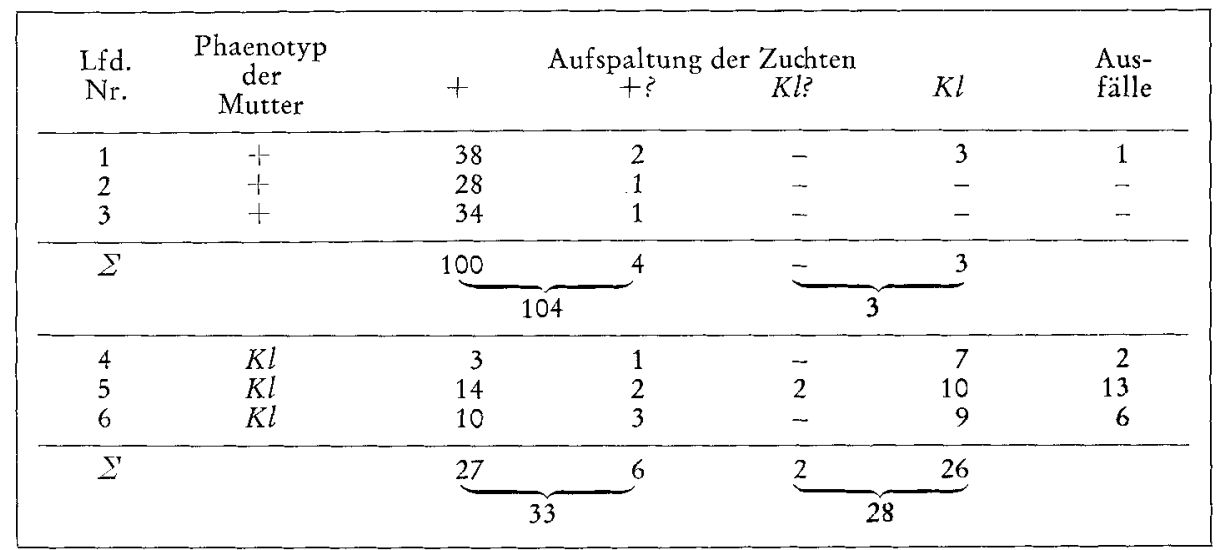

$+/+-$ Kreuzung angesehen werden. Die Kreuzung der $K l-q 9$ mit $\partial \hat{0}$ aus dem Normal-Stamm erbrachte insgesamt 35,2\% $\mathrm{Kl}$-Individuen (Tab. 10, lfd. Nr. 3 bis 7). Sie muß als $K l /+-X+1+-K$ reuzung interpretiert werden. Die starke Verschiebung vom 1:1-Verhältnis läßt sich erklären mit der hohen Zahl an Ausfällen. Kl-Tiere beginnen manchmal sehr spät mit der Eiablage und legen nur wenige Kokons ab. Es ist zu vermuten, daß die Mehrzahl der Tiere, die keine Eier abgelegt haben, in diesen Kreuzungen und im $K l$-Stamm genetisch $K l$-Tiere sind. Manche von ihnen besitzen nicht einmal ein normal ausgebildetes Ovar, wie die Kontrolle der $q$ 우 unter dem Stereo-Mikroskop erkennen ließ.

Ahnlich verhielten sich Kreuzungen mit $q 0$ aus dem $K^{3}{ }^{3}-S t a m m$ (Tabelle 11) mit zwei Abweichungen: (1) Die Aufspaltung der $K l /+-\times+/+-N a c h k o m m e n s c h a f t e n$ nähert sich stärker dem 1:1-Verhältnis (lfd. Nr. 4 bis 6) und (2) in den Nachkommenschaften der + 90 treten unter 107 geprüften weiblichen Nachkommen $3 \mathrm{Kl}$ - $+q$ auf. Diese Ausnahmen können nicht erklärt werden. Eine nur aus $K l$-Tieren bestehende Nachkommenschaft wurde ebenso wenig gefunden wie in den $K L$-Stammzuchten.

Um zu prüfen, ob trotz der Abweichung vom 1:1-Verhältnis ein monohybrider Erbgang vorliegt, wurden Nachzuchten der $K l^{1} /+X+1+-K$ reuzung mit $K l-\hat{\delta} \hat{\delta}$ und $+/+-q 0$ durchgeführt. Die Nachkommenschaften der $K L-$ - $q$ spalteten auf mit einem $K l$-qO-Anteil von insgesamt 43,1\% (Tab. 12, lfd. Nr. 5 bis 7). Die Nachkommenschaften der $+-q Q$ waren dagegen uneinheitlich. Zwei Nachkommenschaften (Tab. 12, lfd. Nr. 1 und 4) bestanden nur aus + -Tieren und einem $K$-Tier (lfd. Nr. 2) ohne jeden Ausfall, so daß hier kein verschobenes 1:1-Verhältnis vorliegen konnte, und eine weitere Nachkommenschaft bestand aus $6+-$ Tieren und $3 \mathrm{Kl}$-Tieren (Ifd. Nr. 3). Daß es sich bei diesen $K l$-Tieren auch genetisch um $K l /+$-Tiere handelt, wird durch eine von zwei Ausnahme-Tieren hervorgebrachte $\mathrm{F}_{3}$ belegt. Beide $\mathrm{F}_{3}-\mathrm{Zuchten}$ spalteten in $\mathrm{Kl}$ und +-Tiere auf (Tab. 12, 1fd. Nr. 8 und 9). Die nächstliegende Erklärung für die Ausnahmen ist die, daß $K l /+-\hat{O} \hat{O}$ wenigstens hin und wieder fertil sein können und an diesen Nachkommenschaften ganz oder teilweise beteiligt waren. Da sich aber die 
Tabile 12

Nachzuchten aus $K l^{1}-q \times+1+-\hat{o}$

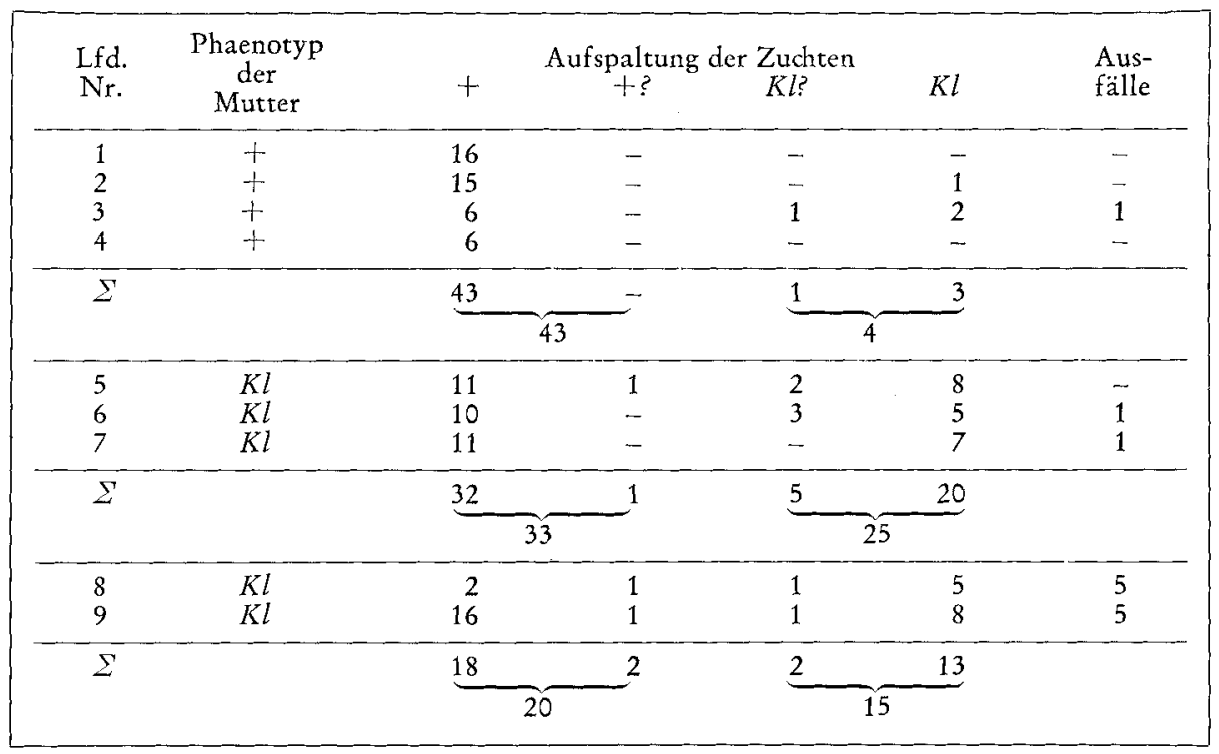

Ausnahme-Kl-우 der Tabelle 11 (lfd. Nr. 1) nicht so erklären lassen, müssen noch andere Entstehungsmöglichkeiten existieren.

Die Übereinstimmung der Kreuzungsergebnisse mit der Erwartung bei einem monohybriden dominanten Erbgang ist ausreichend, wobei folgende Besonderheiten berücksichtigt werden müssen: Die benutzten $K l$-우 sind heterozygot für $K l(K l /+-$ 우). Homozygote $K l / K l$-Tiere wurden weder in den Kreuzungen noch im $K l^{1}$ - oder $K l^{3}$-Stamm angetroffen, sie sind entweder nicht lebensfähig oder aber steril, oder aber sie kommen deswegen nicht zustande, weil $K l /+-\hat{\delta} \delta$ nur geringe Begattungschancen haben. Die Abweichung vom 1:1-Verhältnis in Kreuzungen mit $K l /+-q O+$ erklärt sich durch die verspätete oder vielfach ausbleibende Eiablage der $K l$ - +9 , die dadurch der Zuordnung zur $K l$-Klasse entgehen.

\section{Beziehung zwischen Eigröße und Genotyp}

Die beiden reziproken Kreuzungsserien $G r / G r \times+/+$ und $+/+\times G r / G r$ (Tab. 1 und 2) zeigen, daß die Eigröße nicht durch den Genotyp der eindringenden Spermien beeinflußt wird, sondern nur vom Genotyp der Mutter abhängt. Aus beiden Kreuzungen gehen befruchtete Eier des Genotyps $G r /+$ hervor. Die $\hat{o}$-Eier der $+1+$ Mütter sind dabei normal, die $\hat{\delta}$-Eier der $G r / G r$ - $+q$ groß.

$\mathrm{Zu}$ betrachten wäre noch die Frage nach der Zell-Autonomie von $G r$. In einer $G r /+-$ Mutter könnten die +-Eier normal, die $G r$-Eier größer ausgebildet werden. Diese Möglichkeit hat wenig Wahrscheinlichkeit für sich, da die Reifungsteilungen erst 
nach der Ablage, also längst nach Erreichen der definitiven Eigröße stattfinden. Sie wird völlig ausgeschlossen durch die in Abbildung 3 (Kurve: $G r /+$ ) zusammengetragenen Daten. Die $\mathrm{Gr} /+$ - + +o legen $\delta$-Eier, deren Größenverteilung eindeutig einer eingipfligen Kurve folgt, das gleiche gilt für die Heterozygoten von $K l$ (Abb. 3, Kurve: $K l+$ ).

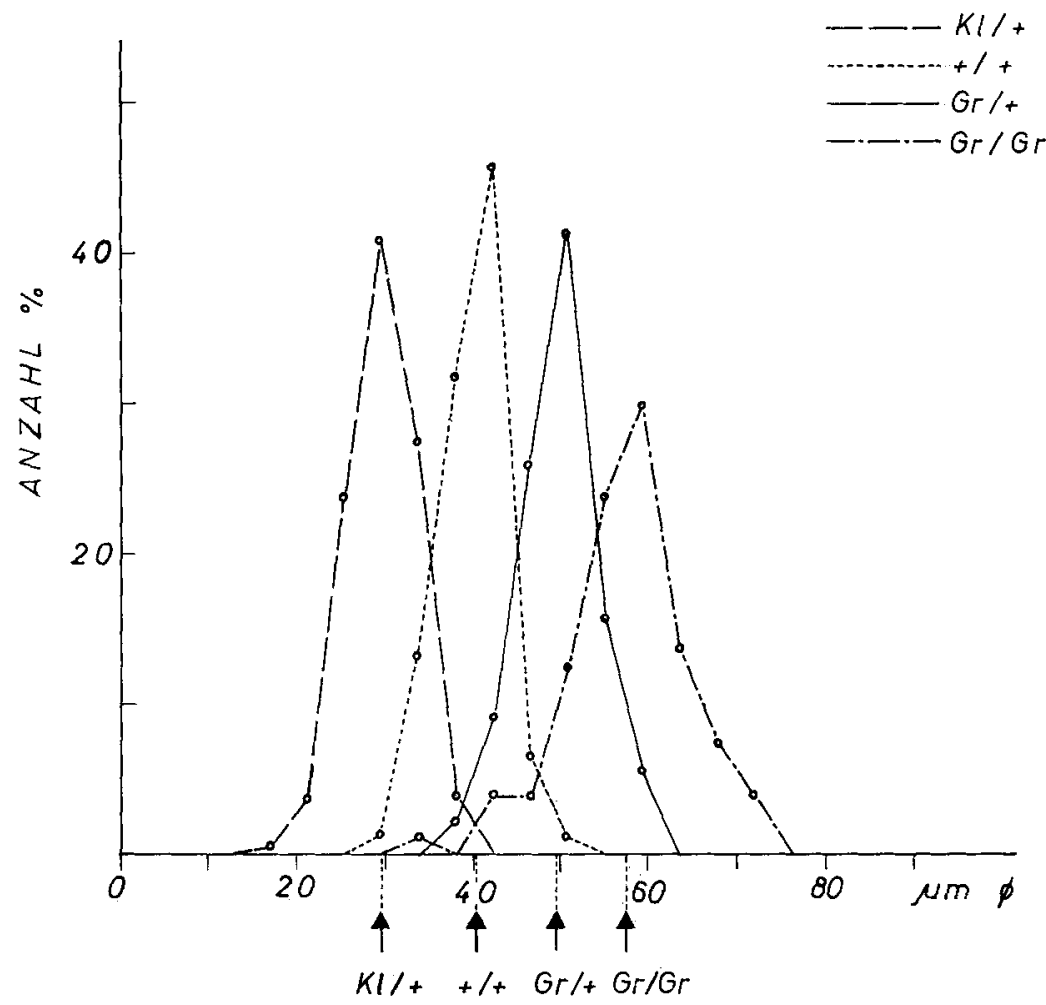

Abb. 3. Verteilung der $\delta$-Ei-Durchmesser von $\mathrm{Kl} /+-,+/+-, \mathrm{Gr} /+$ - und $\mathrm{Gr} / \mathrm{Gr}$-Muttertieren. Gemessen wurde bei jedem Ei der kleinste Durchmesser (Breite). Die Pfeile weisen auf die Mittelwerte der Gruppen hin

So bleibt noch die Frage, ob sich homozygoter und heterozygoter Zustand von Gr-우 unterschiedlich auf die Eigröße auswirken. Zur Prüfung dieser Frage konnten die ausgemessenen Eiablagen der im genetischen Experiment (Tab. 1) getesteten $G r / G r-$ und $G r /+-q Q$ benutzt werden. Ausgenommen wurden nur $q Q$, die versehentlich in ungünstiges Wasser gesetzt worden waren (lfd. Nr. 1 bis 4 sowie 11 und 12). Von den übrigen 우, die unter gleichen äußeren Bedingungen gehalten worden waren, wurden die Größenmessungen in Abbildung 3 zusammengetragen. Aus der Darstellung geht hervor, daß die homozygoten $G r / G r$-oㅇ im Durchschnitt noch größere Eier legen als die heterozygoten $G r /+$-Tiere. Die Differenz der Mittelwerte ist signifikant $(p<0,001)$. Das Merkmal eignet sich aber wegen der starken Uberschneidung nicht für eine saubere Trennung der Genotypen. 
In Abbildung 3 sind neben den $\hat{\sigma}$-Eigrößen aus Ablagen von $G r / G r$ - und $G r /+q q$ auch Messungen an $\delta$-Eiern von $K l 1 /+-q q$ und von $q q$ aus dem NormalStamm zusammengefaßt. Die Darstellung gibt den Größenbereich der $\hat{\delta}$-Eier wieder, die von den $O Q+$ der hier benutzten Genotypen unter vergleichbaren äußeren Bedingungen abgelegt wurden. Diese Genotypen beeinflussen aber nicht nur die $\hat{\jmath}$-Eigröße, sondern auch die Größe der abgelegten Q-Eier, wie aus Tabelle 13 hervorgeht. Die Unterschiede sind allerdings nicht so ins Auge fallend wie die der ô-Eigrößen.

Tabelle 13

Mittlerer Eidurchmesser in Abhängigkeit vom Genotyp des Muttertieres (Stamm AIII)

\begin{tabular}{|ccccc|}
\hline $\begin{array}{c}\text { Genotyp } \\
\text { der } \\
\text { Muitter }\end{array}$ & $\mathrm{n}$ & \multicolumn{2}{c}{-Eier } & \multicolumn{2}{c}{ Q-Eier } \\
\hline$K l++$ & 189 & 29,7 & $\mathrm{n}$ & $\mu \mathrm{m} \varnothing$ \\
$+l+$ & 151 & 40,1 & 125 & 77,5 \\
$G r /+$ & 128 & 49,8 & 320 & 80,6 \\
$G r / G r$ & 177 & 57,5 & 1352 & 84,8 \\
\hline
\end{tabular}

Tabelle 14

Mirtlerer Eidurchmesser in Abhängigkeit von der Zuchttemperatur. Ablagen vom 4. bis 9. Tag nach Überführen in die Versuchsbedingungen (Stamm AIII [normal])

\begin{tabular}{|cccccc|}
\hline $\begin{array}{c}\text { Zucht- } \\
\text { temperatur }\end{array}$ & $\mathrm{n}$ & $\delta$-Eier & $\mu \mathrm{m} \varnothing$ & $\mathrm{n}$ & \multicolumn{2}{c}{ Q-Eier } \\
\hline $16^{0} \mathrm{C}$ & 104 & 42,2 & 169 & 79,8 \\
$20^{\circ} \mathrm{C}$ & 154 & 39,6 & 200 & 76,9 \\
$26^{\circ} \mathrm{C}$ & 276 & 38,4 & 294 & 76,3 \\
\hline
\end{tabular}

Die Eigröße ist aber nicht nur vom Genotyp der Mutter abhängig, sondern auch von verschiedenen äußeren Bedingungen. So ändert sich die Größe der abgelegten Eier

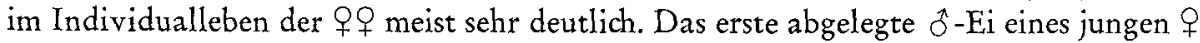
ist fast immer deutlich kleiner als die folgenden. Bei den +-OPQ beträgt der Durchmesser des ersten abgelegten $\hat{\delta}$-Eies meist 30 bis $34 \mu \mathrm{m}$, während die folgenden einen Durchmesser von 38 bis $42 \mu \mathrm{m}$ haben. Die $G r$-웅 beginnen ihre Ablage meist mit einem $\hat{\partial}-\mathrm{Ei}$ von 38 bis $42 \mu \mathrm{m}$ Durchmesser und legen später $\hat{\delta}$-Eier mit einem Durchmesser von etwa 47 bis $60 \mu \mathrm{m}$.

Ein Einfluß der Temperatur auf die Eigröße wurde in einem Experiment untersucht, dessen Ergebnisse in Tabelle 14 dargestellt worden sind. Gleichaltrige begattete $+1+-q q$ wurden 3 Tage nach Ablagebeginn in 3 Gruppen von je $16 q q$ aufgeteilt. Die eine Gruppe wurde weiterhin unter Normalbedingungen $\left(20^{\circ} \mathrm{C}\right)$ gehalten, die zweite Gruppe wurde bei $16^{\circ} \mathrm{C}$ und die dritte bei $26^{\circ} \mathrm{C}$ weitergezüchtet. In Tabelle 14 sind die durchschnittlichen Eidurchmesser der am 4. bis 9. Tag nach Uberführung in die Versuchsbedingungen abgelegten Eier dargestellt. Die Varianzanalyse mit anschließendem Duncan-Test ergab signifikante Differenzen $(p \ll 0,001)$ zwischen allen Mittelwerten mit Ausnahme der Differenz der $q$-Ei-Durchmesser bei $20^{\circ} \mathrm{C}$ und $26^{\circ} \mathrm{C}(p \approx 0,7)$. 


\section{DISKUSSION}

Im folgenden soll nur die Frage nach dem Status der $G r$ - und $K l$-Mutation geprüft werden. Uberraschend ist, daß anscheinend alle gefundenen Mutanten-Typen mehrfacb auftreten. "Gr" trat zweimal auf, „Kl" siebenmal und „Gr $z u+$ " in einer Häufigkeit von 1,4 bis $5,0 \%$. Die später verlorengegangene zweite $G r$-Mutante zeigte insofern formale Ubereinstimmung mit der analysierten $G r$-Mutante, als sie ebenfalls dominant vererbt wurde. Eine gleichfalls formale Übereinstimmung untereinander zeigen auch die beiden geprüften $K l$-Mutanten. Eine Reihe weiterer $K l$-Mutanten konnte nicht untersucht werden. Es ist zu fragen, ob diese formalen Ubereinstimmungen auf einer genetischen Identität beruhen.

Am besten läßt sich die Mutation „Gr zu +" untersuchen, die sich in der hohen Rate an Wildtyp-Ausnahmetieren in Kreuzungen mit homozygoten $G r$-Tieren zeigt. Sie beträgt 5,0\% in den $G r / G r-X+/+-K r e u z u n g e n$ und $1,4 \%$ in den $+/+-\times G r / G r-$ Kreuzungen. Der Unterschied in der Häufigkeit der Ausnahmetiere zwischen den reziproken Kreuzungen ist statistisch gesichert $(0,005>p>0,001)$, wenn man die + ?Tiere zu den +-Tieren zählt. Die Differenz ist nicht gesichert $(0,1<p<0,2)$, wenn man alle + ?-Tiere in Wirklichkeit zu den $G r$-Tieren zu zählen hat. Aber selbst wenn man nur die Zahl der sicheren +-Tiere berücksichtigt, ist der Anteil der Ausnahmetiere mit 3,2\% und $1,4 \%$ in den reziproken Kreuzungen noch überraschend hoch. Die + Ausnahmetiere waren mit $+/+-\hat{\delta} \hat{\delta}$ aus dem Normal-Stamm gekreuzt worden und hatten sich dabei genetisch als $+/+-$ Tiere erwiesen. Man kann die +- Ausnahmetiere also als Rückmutanten zum Wildtyp betrachten. Diese Aussage muß aber angezweifelt werden wegen der Seltenheit, mit der Rückmutationen im allgemeinen erfolgen (TIMoFÉEFF-Ressovsky 1937, GREEN 1957). Bei viclen Rückmutationen handelt es sich darüber hinaus nicht um „echte“ Rückmutationen zum Wildtyp, sondern nur um „funktionelle" Rückmutationen, die durch kompensierende Suppressor-Mutationen an anderen Genloci vorgetäuscht werden (YANOFSKY \&LAwRENCE 1960). Eine solche Möglichkeit kann für alle Loci außerhalb der $G r$-Kopplungsgruppe auf Grund der Kreuzungen Ausnahme-o우 $X+1+-\hat{\delta} \hat{\delta}$ ausgeschlossen werden. Die Nachkommenschaften dieser Kreuzungen bestanden nur aus +- Tieren. Nicht auszuschließen ist eine kompensierende Mutation innerhalb der $G r$-Kopplungsgruppe. Neben der ungewöhnlichen Häufigkeit, mit der ein solcher Schritt erfolgen müßte, ist aber auch noch die unterschiedliche Häufigkeit $z$ wischen "Hinmutationen" ( 1 bis 2 unter 3500 ) und Rückmutationen $(1,4$ bis 5,0\%) kritisch zu betrachten. Zwei Interpretationsmöglichkeiten bieten sich an: es könnte sich bei $G r$ um ein instabiles Gen (Demerec 1935, McCurntock 1956) handeln oder um eine Chromosomenmutation oder Chromosomenaberration, für die relativ hohe Raten nachgewiesen wurden (RIEGER 1963, ZELENY 1921). Die Rückmutationen können bei dieser Vorstellung tatsächlich als Rückkehr zum Wildtyp betrachtet werden. Für diese Interpretation spräche auch das doppelte Auftreten von $G r$ und das mehrfache Auftreten von $K l$ nur in $G r$-Nachkommenschaften. $K l$ wäre dann ebenfalls als Chromosomenmutation oder Chromosomenaberration zu betrachten, und man könnte vermuten, daß der formalen Übereinstimmung der $K l$-Mutanten untereinander eine genetische Übereinstimmung zugrunde liegt.

Eine sehr gute Übereinstimmung mit den gefundenen Daten würde eine Deutung 
von $G r$ als einer Chromosomenaberration in der Art der Trisomie bieten. Die Homozygoten wären dann als tetrasom aufzufassen, und die hohe Rückmutationsrate würde sich aus den Verteilungsschwierigkeiten bei der Meiosis erklären, in deren Gefolge relativ oft euploide Gameten entstehen müßten. Um die Richtigkeit einer solchen Interpretation abzusichern, müssen aber noch genauere Karyotyp-Untersuchungen oder aber Analysen mit neuen Markiergenen derselben Kopplungsgruppe abgewartet werden.

Um die Beziehungen zwischen $K l$ und $G r$ zu untersuchen, wurden Kreuzungen zwischen Tieren der $K l$ - und $G r$-Stämme angesetzt. Besonderer Wert wurde darauf gelegt, unter den Nachkommen doppelt heterozygote $q O$ durch Rückkreuzungen mit $+1+-\hat{o} \hat{o} \mathrm{zu}$ identifizieren. In den noch nicht abgeschlossenen Versuchen ließen sich bisher keine Rekombinanten von $G r$ und $K l$ nachweisen. Diese Kombination ist demnach entweder letal oder aber $G r$ und $K l$ interferieren in anderer Weise miteinander.

\section{ZUSAMMENFASSUNG}

1. Der marine Archiannelide Dinophilus gyrociliatus besitzt einen extremen Geschlechtsdimorphismus. Auch die Eier, aus denen 우우 und $\delta \hat{\delta}$ hervorgehen, haben schon eine unterschiedliche Größe. In der vorliegenden Arbeit wird die Kreuzungsund Zuchttechnik für genetisches Arbeiten mit Dinophilus und das Erbverhalten zweier Mutanten beschrieben.

2. Charakteristisches Merkmal der in Laborzuchten aufgetretenen Mutanten $K l$ und $G r$ ist eine gegenüber der Norm verringerte $(K l)$ bzw. erhöhte $(G r)$ Größe der $\hat{\delta}$-Eier. Beide Mutanten zeigen einen monohybriden Erbgang mit Dominanz von $K l$ bzw. $G r$ gegenüber $+K l$ bzw. $+G r$.

3. Entscheidend für die Eigröße ist der Genotyp des Muttertieres, nicht der des Eies.

4. In der Kreuzung $+1+\times G r /+$ ist der Anteil der $G r /+-N a c h k o m m e n$ kleiner als 0,5. Die Gr-Spermien besitzen offenbar eine geringere Befruchtungschance als die + -Spermien.

5. $G r /+-\hat{o} \hat{o}$ und $G r / G r-\hat{o} \hat{o}$ bringen neben normalen Spermien solche mit 2 und 4 Schwänzen hervor. Ein Zusammenhang mit der geringeren Befruchtungshäufigkeit durch $G r$-Spermien ließ sich nicht ermitteln.

6. In den Kreuzungen $+1+\times G r / G r$ und $G r / G r \times+1+$ treten 1,4 bis $5,0 \%$ Wildtyp-Nachkommen auf, die sich auch genetisch wie $+/+$-Tiere verhalten und daher als Rückmutanten zum Wildtyp angesehen werden müssen.

7. Gr läßt sich als instabiles Gen oder wahrscheinlicher als Chromosomenmutation bzw. Chromosomenaberration interpretieren.

Dem Direktor des Zoologischen Instituts der Universität des Saarlandes, Herrn Professor Dr. G. DE LatTin danke ich für die freundliche Unterstützung der Arbeit. Für ihre Hilfe bei der Zucht und den Messungen bin ich Frau cand. rer. nat. H. Scharwath und Hern cand. rer. nat. H.-J. LANG sehr dankbar. 


\section{ZITIERTE LITERATUR}

Demerec, M., 1935. Unstable genes. Bot. Rev. 1, 233-248.

Green, M. M., 1957. Reverse mutation in Drosophila and the status of the particulate gene. Genetica 29, 1-38.

Korschelt, E., 1882. Uber Bau und Entwicklung von Dinophilus apatris. Z. wiss. Zool. 37, 315-353.

McClintock, B., 1956. Intranuclear systems controlling gene action and mutation. Brookhaven Symp. Biol. 8, 58-74.

RIEGER, R., 1963. Die Genommutationen (Ploidiemutationen). Jena, G. Fischer, 183 pp.

TIMofÉEFF-RessovsKY, N. W., 1937. Experimentelle Mutationsforschung in der Vererbungslehre. Dresden, Steinkopff, $181 \mathrm{pp}$.

Traut, W., 1966a. Eine Mutante mit vergrößerten Männchen-Eiern bei Dinopbilus gyrociliatus (Archiannelida). Experientia 22, 237-238.

- 1966b. Über die Kopulation bei Dinopbilus gyrociliatus (Archiannelida). Zool. Anz. 177, 402-411.

- 1967. Uber die Geschlechtsbestimmung bei Dinopbilus gyrociliatus (Archiannelida). Habil.Schr, Saarbrücken.

YanOFSKy, C. \& Lawrence, P. ST., 1960. Gene action. A. Rev. Microbiol. 14, 311-340

ZELENY, C, 1921. The direction and frequency of mutation in the bar-eye series of multiple allelomorphs of Drosopbila. J. exp. Zool. 34, 203-233. 